\title{
Mobile technology for self-monitoring of blood glucose among patients with type 2 diabetes mellitus
}

\author{
Steven S. Coughlin ${ }^{1,2}$ \\ ${ }^{1}$ Department of Clinical and Digital Health Sciences, College of Allied Health Sciences, Augusta University, Augusta, GA, USA; ${ }^{2}$ Charlie Norwood \\ Veterans Affairs Medical Center, Augusta, GA, USA \\ Correspondence to: Dr. Steven S. Coughlin, PhD. Associate Professor, Department of Clinical and Digital Health Sciences, College of Allied Health \\ Sciences, Augusta University, $112015^{\text {th }}$ Street, EC-4324, Augusta, GA 30912, USA. Email: scoughlin@augusta.edu. \\ Provenance: This is an invited Editorial commissioned by Section Editor Dr. Kaiping Zhang, PhD (AME College, AME Group, China). \\ Comment on: Hu ZD, Zhang KP, Huang Y, et al. Compliance to self-monitoring of blood glucose among patients with type 2 diabetes mellitus and its \\ influential factors: a real-world cross-sectional study based on the Tencent TDF-I blood glucose monitoring platform. Mhealth $2017 ; 3: 25$.
}

Received: 29 August 2017; Accepted: 10 October 2017; Published: 30 October 2017.

doi: $10.21037 /$ mhealth.2017.10.03

View this article at: http://dx.doi.org/10.21037/mhealth.2017.10.03

Worldwide, the number of people with diabetes is increasing and about $90 \%$ of patients have type 2 diabetes mellitus; about one fifth of people with type 2 diabetes are on insulin treatment (1). The global burden of type 2 diabetes has prompted increasing efforts to develop mobile technologies for self-monitoring of blood glucose among patients with diabetes. A wide variety of home glucometers are available that are portable, inexpensive, reliable and sensitive, and which use smaller amounts of blood than in the past. After washing their hands and using alcohol wipes, patients use a lancet to obtain a tiny amount of blood from a fingertip and place it on or beside a test strip placed in a glucometer. Recent developments include the introduction of mobile devices for self-monitoring of blood glucose that are coupled with a smartphone app that communicates test results to providers $(2,3)$, bloodfree mobile devices for measuring blood glucose that use a sensor placed on the forearm (1), and devices for the continuous monitoring of blood glucose in patients with insulin-dependent diabetes (4).

The American Diabetes Association recommends that patients whose medication regiment includes multiple daily insulin injections or insulin pumps should test their blood glucose at least three times per day (5). Although self-monitoring of blood glucose can be a useful tool for decision-making by patients and clinicians, there has been controversy over the value of self-monitoring of blood glucose by diabetic patients who are not receiving insulin (6). For example, a recent pragmatic, randomized controlled trial of glucose self-monitoring in non-insulin using patients with type 2 diabetes in primary care settings found no significant differences in hemoglobin A1c (HgbA1c) levels or health-related quality of life across groups (7). Malanda et al. (8) conducted a Cochrane database systematic review of randomized controlled trials of self-monitoring of blood glucose in patients with type 2 diabetes mellitus who were not using insulin. Twelve randomized controlled trials were included and evaluated outcomes in 3,259 patients. The results indicated that when the duration of diabetes is over one year, the overall effect of self-monitoring of blood glucose on glycemic control in patients with type 2 diabetes who are not using insulin is small up to six months after initiation and subsides after 12 months. Further, there was no evidence that self-monitoring of blood glucose affects patient satisfaction, general well-being, or general health-related quality of life (8). More recently, Mannucci (9) conducted a meta-analysis of eight randomized controlled trials of the use of self-monitoring of blood glucose in patients with non-insulin-treated type 2 diabetes compared with no self-monitoring (1,277 and 1,072 patients, respectively). Self-monitoring of blood glucose was found to reduce HgbA1c by $-0.17 \%$ [95\% confidence interval (CI): $-0.25 \%$ to $-0.09 \%, \mathrm{P}<0.003]$. The reduction in $\mathrm{HgbA1c}$ was greater in randomized controlled trials $(n=3)$ in which self-monitoring blood glucose data were used to adjust 
diabetes medications [HgbA1c $-0.1 \%$ (95\% CI: $-0.49 \%$ to $-0.1 \%)$ ] than in randomized controlled trials in which self-monitoring blood glucose data were not used for that purpose [HbgA1c decrease $-0.3 \%$ (95\% CI: $-0.2 \%$ to $0.0 \%), \mathrm{P}<0.005]$. Zhu et al. (10) conducted a metaanalysis of 15 randomized controlled trials of the use of self-monitoring of blood glucose in patients with noninsulin-treated type 2 diabetes (3,383 patients). The results indicated that self-monitoring of blood glucose improved HgbA1c (mean difference $-0.33,95 \%$ CI: -0.45 to $-0.22, \mathrm{P}=0.00000003)$, body mass index $(-0.65,95 \%$ CI: -1.18 to $-0.12, \mathrm{P}=0.0164)$, and total cholesterol $(-0.12$, $95 \%$ CI: -0.20 to $-0.04, \mathrm{P}=0.0034)$ more effectively than the control. In addition, self-monitoring of blood glucose improved HgbA1c levels in the short term $(<6$ months follow-up) and long term (>12-month follow-up) in patients with type 2 diabetes who were not using insulin. A subgroup analysis of geographical region of the trials showed significant decreases in HgbA1c levels in the Asia and America-Europe groups (10).

A potentially important development in diabetes care is the introduction of telehealth remote monitoring for patients with type 2 diabetes who perform self-monitoring of blood glucose (11). Telehealth remote monitoring, which may improve clinical outcomes, care coordination, and patient satisfaction, allows for patients to inform their health care providers about glycemic control and for diabetes educators or other providers to monitor glucose test results and to provide feedback to patients in real-time to increase knowledge, self-efficacy, and behavior change (12-15). This feedback loop of physiologic data collection and interpretation combined with feedback to the patient allows for modified treatment plan by health care providers and improved outcomes.

In this issue of the journal, $\mathrm{Hu}$ et al. (16) report results of a cross-sectional study of use of the Tencent TDF-I (Tencent, Beijing, China) blood glucose monitoring platform by 415 Chinese patients with type 2 diabetes mellitus. The study lacked a comparison group and was limited by the non-randomized controlled design and short observation period (90 days after enrollment). The study by $\mathrm{Hu}$ et al. (16) does not provide any information about the effectiveness of the Tencent TDF-I blood glucose monitoring platform relative to other devices for home measurement of blood glucose. Comparative studies are needed that examine the effectiveness of mobile technologies for improving diabetes care among patients with diabetes, especially studies with a rigorous study design and longer durations of follow-up. Studies that examine the effectiveness of new technologies for self-monitoring of blood glucose can use a usual care comparison group. Equivalency trials that test the relative effectiveness of two or more mobile technologies for improving diabetes care are also of interest. There is also a need for cost-effectiveness studies of mobile technologies for self-monitoring of blood glucose (17).

Of particular concern is the accuracy and precision of mobile devices for monitoring blood glucose (18-21). Self-monitoring of blood glucose is a key tool for the management of insulin-dependent diabetes and adjustment of insulin therapy. Inaccuracies and a lack of precision of mobile devices impair the detection of hypoglycemia and glucose variability (1). Mobile devices for continuous glucose monitoring require repeated calibration.

Many counties regulate mobile technologies for improving disease management. The U.S. Food and Drug Administration (FDA) draws a distinction between unregulated apps and mobile medical devices and apps that are subject to overt FDA regulation and unregulated apps such as those used by consumers for diabetes selfmanagement (22). Apps that convert a mobile platform such as a smartphone or tablet computers into a medical device are regulated by the FDA. The FDA regulates mobile apps that pose a greater risk to patients if they do not function as intended (e.g., apps that perform clinical tests such as analysis of blood glucose). Many unregulated smartphone apps are sold directly to consumers to help people with diabetes to control their fitness and health through diabetes education, healthy diet and nutrition, physical activity, weight management, and monitoring blood glucose levels and medications $(23,24)$. However, a recent review of smartphone apps for diabetes self-management available from Google Play Store and Windows Phone Store found that 56 of 65 apps did not meet minimal requirements or did not work properly (24). Nie et al. (23) evaluated 95 Chinese apps for diabetes self-management and found that few allowed patients to track their blood pressure (14\%), cholesterol (14\%), or body mass index (11\%).

In conclusion, self-monitoring of blood glucose is a mainstay of diabetes management, especially for patients receiving insulin therapy, which allows patients to become engaged in their own care (25). Before such devices are widely introduced, the effectiveness of mobile technologies for self-monitoring of blood glucose by diabetic patients should be examined in carefully designed randomized controlled trials. Information about the accuracy, precision, 
safety, and cost-effectiveness of these mobile devices is also important.

\section{Acknowledgements}

None.

\section{Footnote}

Conflicts of Interest: The author has no conflicts of interest to declare.

Disclaimer: the contents do not represent the views of the U.S. Department of Veterans Affairs or the U.S. Government.

\section{References}

1. Haak T, Hanaire H, Ajjan R, et al. Flash glucose-sensing technology as a replacement for blood glucose monitoring for the management of insulin-treated type 2 diabetes: a multicenter, open-label randomized controlled trial. Diabetes Ther 2017;8:55-73.

2. Garg SK, Shah VN, Akturk HK, et al. Role of mobile technology to improve diabetes care in adults with type 1 diabetes: the remote-T1D Study iBGStar in type 1 diabetes management. Diabetes Ther 2017;8:811-9.

3. Jendrike N, Baumstark A, Chen CH, et al. Introduction of a novel smartphone-coupled blood glucose monitoring system. J Diabetes Sci Technol 2017. [Epub ahead of print].

4. Carlson AL, Mullen DM, Bergenstal RM. Clinical use of continuous glucose monitoring in adults with type 2 diabetes. Diabetes Technol Ther 2017;19:S4-11.

5. American Diabetes Association. Approaches to glycemic treatment. Sec. 7. In Standards of Medical Care in Diabetes-2015. Diabetes Care 2015;38:S41-8.

6. Davidson MB. Counterpoint: self-monitoring of blood glucose in type 2 diabetic patients not receiving insulin: a waste of money. Diabetes Care 2005:28:1531-3.

7. Young LA, Buse JB, Weaver MA, et al. Glucose selfmonitoring in non-insulin-treated patients with type 2 diabetes in primary care settings. A randomized trial. JAMA Intern Med 2017;177:920-9.

8. Malanda UL, Welschen LM, Riphagen II, et al. Selfmonitoring of blood glucose in patients with type 2 diabetes mellitus who are not using insulin. Cochrane Database Syst Rev 2012;1:CD005060.

9. Mannucci E, Antenore A, Giorgino F, et al. Effects of structured versus unstructured self-monitoring of blood glucose on glucose control in patients with non-insulintreated type 2 diabetes: a meta-analysis of randomized controlled trials. J Diabetes Sci Technol 2017. [Epub ahead of print].

10. Zhu H, Zhu Y, Leung SW. Is self-monitoring of blood glucose effective in improving glycaemic control in type 2 diabetes without insulin treatment: a meta-analysis of randomised controlled trials. BMJ Open 2016;6:e010524.

11. Greenwood DA, Blozis SA, Young HM, et al. Overcoming clinical inertia: a randomized clinical trial of a telehealth remote monitoring intervention using paired glucose testing in adults with type 2 diabetes. J Med Internet Res 2015;17:e178.

12. Shea S, Weinstock RS, Starren J, et al. A randomized trial comparing telemedicine case management with usual care in older, ethnically diverse, medically underserved patients with diabetes mellitus. J Am Med Inform Assoc 2006;13:40-51.

13. Darkins A, Ryan P, Kobb R, et al. Care Coordination/ Home Telehealth: the systematic implementation of health informatics, home telehealth, and disease management to support the care of veteran patients with chronic conditions. Telemed J E Health 2008;14:1118-26.

14. Ceriello A, Barkai L, Christiansen JS, et al. Diabetes as a case study of chronic disease management with a personalized approach: the role of a structured feedback loop. Diabetes Res Clin Pract 2012;98:5-10.

15. Greenwood DA, Young HM, Quinn CC. Telehealth remote monitoring systematic review: structured selfmonitoring of blood glucose and impact on A1c. J Diabetes Sci Technol 2014;8:378-89.

16. Hu ZD, Zhang KP, Huang Y, et al. Compliance to selfmonitoring of blood glucose among patients with type 2 diabetes mellitus and its influential factors: a real-world cross-sectional study based on the Tencent TDF-I blood glucose monitoring platform. mHealth 2017;3:25.

17. Chaugule S, Graham C. Cost-effectiveness of G5 Mobile continuous glucose monitoring device compared to selfmonitoring of blood glucose alone for people with type 1 diabetes from the Canadian societal perspective. J Med Econ 2017;11:1-8.

18. Link M, Schmid C, Pleus S, et al. System accuracy evaluation of four systems for self-monitoring of blood glucose following ISO 15197 using a glucose oxidase and a hexokinase-based comparison method. J Diabetes Sci Technol 2015;9:1041-50.

19. Baumstark A, Jendrike N, Pleus S, et al. Evaluation 
of accuracy of six blood glucose monitoring systems and modeling of possibly related insulin dosing errors. Diabetes Technol Ther 2017. [Epub ahead of print].

20. Bailey TS, Wallace JF, Pardo S, et al. Accuracy and user performance evaluation of a new, wireless-enabled blood glucose monitoring system that links to a smart mobile device. J Diabetes Sci Technol 2017;11:736-43.

21. Christiansen M, Greene C, Pardo S, et al. A new, wirelessenabled blood glucose monitoring system that links to a smart mobile device: accuracy and user performance evaluation. J Diabetes Sci Technol 2017;11:567-73.

22. Coughlin S, Thind H, Liu B, et al. Mobile phone apps for preventing cancer through educational and behavioral

doi: $10.21037 /$ mhealth.2017.10.03

Cite this article as: Coughlin SS. Mobile technology for self-monitoring of blood glucose among patients with type 2 diabetes mellitus. mHealth 2017;3:47. inteventions: state of the art and remaining challenges. JMIR mHealth and uHealth 2016;4:e69.

23. Nie L, Xie B, Yang Y, et al. Characteristics of Chinese $\mathrm{m}$-Health applications for diabetes self-management. Telemed J E Health 2016;22:614-9.

24. Brzan PP, Rotman E, Pajnkihar M, et al. Mobile applications for control and self management of diabetes; a systematic review. J Med Syst 2016;40:210.

25. Kosar L, Alsabbagh W, Lu X, et al. Self-monitoring blood glucose test strip utilization in Saskatchewan: a retrospective study. Can J Diabetes 2017. [Epub ahead of print]. 